\title{
Tractography of Persistent Ipsilateral Hemiparesis Following Subdural Hematoma
}

\author{
Seong-il Oh, Mi-Jung Kim, Kyung-Pil Oh, Hyun Young Kim, Seung Hyun Kim, Hee-Jin Kim
}

Can J Neurol Sci. 2013; 40: 601-602

Ipsilateral hemiparesis is occasionally caused by a medullary lesion or by secondary damage to the cerebral peduncle contralateral to the lesion, a so-called Kernohan-Woltman notch phenomenon (KWNP). A possible mechanism of ipsilateral hemiparesis is that morphological changes in the brainstem associated with KWNP are accompanied by irreversible damage to the adjacent corticospinal tract $(\mathrm{CST}) \cdot{ }^{1-3} \mathrm{~A}$ few reports dealing with the acute and sub-acute stages of this paradoxical phenomenon have appeared in the literature. ${ }^{1,2,4,5}$

In the case reported here, the patient presented with longstanding ipsilateral hemiparesis following traumatic subdural hematoma 16 years previous. We investigated and visualized using fiber tractography (FT) permanent reconfiguration of the CST contralateral to the lesioned side with midbrain atrophy.

\section{Case Report}

A 55-year-old male with right hemiparesis visited the neurology clinic. His medical records revealed that he had been admitted with stuporous mentality after a head trauma 16 years previous. At that time he had a Glasgow Coma Scale of 14 with quadriparesis. Brain computed tomography (CT) showed a large acute subdural hematoma in the fronto-temporo-parietal area of the right hemisphere with midline shift. Decompressive craniectomy with hematoma removal was performed. Although he gradually recovered motor power in the left extremity, right hemiparesis was sustained. He was discharged with a residual right hemiparesis and revisited the hospital due to medical insurance requirements 16 years afterward. He complained of dysarthria and spastic hemiparesis. On neurological examination, the motor strengths of the right extremities were a grade 3 on the Medical Research Council (MRC) scale. Deep tendon reflexes were hyperactive in the right extremities. There were no symptoms related to any of the sensory modalities.

Conventional magnetic resonance (MR) images as well as diffusion tensor images (DTIs) were obtained with a 3.0 T MR imaging scanner (Philips Real Time Compact Magnet 3.0-Tesla MRI system, Achieva 3.0-Tesla X-series) using an 8-element phased-array neurovascular array coil. Fluid-attenuated inversion recovery (FLAIR) images showed asymmetric shrinkage of the right hemisphere and ventricular enlargement in the temporal horn (Figure 1A). Asymmetric loss of volume of the left cerebral peduncle in the midbrain was also apparent (Figure 1B). Diffusion tensor images were obtained using a sequence of single-shot spin-echo-type echo-planar images (TR/TE, 13891/55ms; matrix size, 112×112; FOV, $224 \mathrm{~mm}$; 90 axial sections; $2.0-\mathrm{mm}$ section thickness). At the level of the supratentorial CST on the lateral view, significant degenerated
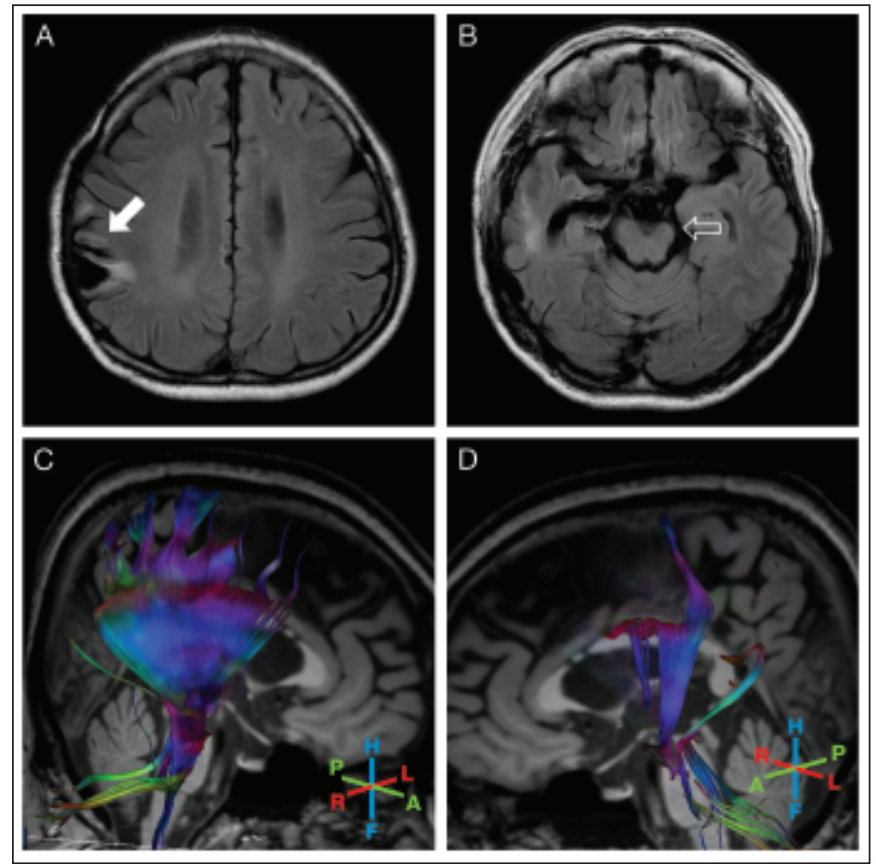

Figure: Brain MRI and fiber tractography. (A) FLAIR images show asymmetric shrinkage of the right hemisphere and ventricular enlargement in the temporal horn (arrow). (B) Mild asymmetric loss of volume of the left cerebral peduncle in the midbrain can be seen (open arrow). $(C, D)$ Fiber tractography of the corticospinal fiber from the lateral view (C: right lateral view; D: left lateral view). The corticospinal fiber in the tractography of the left hemisphere shows severe degeneration $(D)$, while the corticospinal fiber of the right hemisphere is intact $(C)$.

CSTs were observed in the left side, while those of the right side were intact (Figure 1C, D).

\section{Discussion}

Kernohan-Woltman notch phenomenon, which was first described by Kernohan and Woltman, refers to the paradoxical association between an ipsilateral motor deficit and deformation

From the Department of Neurology (SIO, KPO, HYK, SHK, HJK), Rehabilitation Medicine (MJK), College of Medicine, Hanyang University, Seoul, Republic of Korea. Received October 12, 2012. Final Revisions Submitted January 2, 2013. Correspondence to: Hee-Jin Kim, Department of Neurology, College of Medicine, Hanyang University, 17 Haengdang-dong, Seongdong-gu, Seoul, 133-791, Republic of Korea. Email address: hyumcbrain@hanyang.ac.kr. 
in the contralateral cerebral peduncle. ${ }^{3}$ A wide range of serious injuries can increase intracranial pressure and cause development of Kernohan's notch. The morphological changes of KWNP may correlate with indentation of the cerebral peduncle caused by midbrain compression or irreversible tissue damage to the CST in the cerebral peduncle. In the acute and subacute stages of KWNP, ipsilateral hemiparesis may be due to pathological mechanisms, e.g. compressive forces that cause the herniating midbrain to impinge on the contralateral edge of the tentorium cerebelli, contralateral contusion, pressure necrosis secondary to elevated intracranial pressure, or arterial infarction. ${ }^{1-4}$

In this case, after the primary lesion was relieved, degeneration of the CST contralateral to the cerebral peduncle may have been sustained in the compressed free tentorial notch due to the characteristics of the CST, a discrete and compact collection of a large number of axons; interestingly, in the present patient, these axons may have extended for more than a meter from their cell bodies. Another possible mechanism of persistent disruption to the myelinated fibers of the CST in KWNP could be failure of compensatory fiber regeneration.

Fiber tractography revealed that the CST of the right hemisphere was intact, while long-term Wallerian degeneration, potentially caused by the pressure effect of the subdural hematoma, not by parenchymal damage, as well as midbrain atrophy, was observed in the left hemisphere.

In summary, fiber tractography suggested a good diagnostic evidence for investigating the possible mechanism of uncommonly consolidated features of ipsilateral hemiparesis and the distribution of degenerated fibers.

\section{ACKNOWLEDGEMENTS}

Authors Seong-il Oh and Mi-Jung Kim contributed equally to this work.

This study was supported by the cluster research fund of Hanyang University (HY-2009-C). There is no conflicts of interest in this study.

\section{REFERENCES}

1. Jones KM, Seeger JF, Yoshino MT. Ipsilateral motor deficit resulting from a subdural hematoma and a Kernohan notch. AJNR Am J Neuroradiol. 1991;12(6):1238-9.

2. Moon KS, Lee JK, Joo SP, et al. Kernohan's notch phenomenon in chronic subdural hematoma: MRI findings. J Clin Neurosci. 2007;14(10):989-92.

3. Zafonte RD, Lee CY. Kernohan-Woltman notch phenomenon: an unusual cause of ipsilateral motor deficit. Arch Phys Med Rehabil. 1997;78(5):543-5.

4. Carrasco R, Pascual JM, Navas M, Martinez-Florez P, ManzanaresSoler R, Sola RG. Kernohan-Woltman notch phenomenon caused by an acute subdural hematoma. J Clin Neurosci. 2009; 16(12):1628-31.

5. Yamasaki F, Kodama Y, Hotta T, Yuki K, Taniguchi E, Hashizume A. [Magnetic resonance imaging of Kernohan's notch in chronic subdural hematoma]. No To Shinkei. 1997;49(6):563-6. 